\title{
Modulation of Parietal Activation by Semantic Distance in a Number Comparison Task
}

\author{
Philippe Pinel,* Stanislas Dehaene,*,1 Denis Rivière,* and Denis LeBihan† \\ *Unité INSERM 334 and TUNAF, IFR 49, Service Hospitalier Frédéric J oliot, CEAIDSV, \\ 4 Place du Général Lederc, 91401 Orsay Cedex, France
}

Received February 21, 2001

The time to compare two numbers shows additive effects of number notation and of semantic distance, suggesting that the comparison task can be decomposed into distinct stages of identification and semantic processing. Using event-related fMRI and high-density ERPs, we isolated cerebral areas where activation was influenced by input notation (verbal or Arabic notation). The bilateral extrastriate cortices and a left precentral region were more activated during verbal than during Arabic stimulation, while the right fusiform gyrus and a set of bilateral inferoparietal and frontal regions were more activated during Arabic than during verbal stimulation. We also identified areas that were influenced solely by the semantic content of the stimuli (numerical distance between numbers to be compared) independent of the input notation. Activation tightly correlated with numerical distance was observed mainly in a group of parietal areas distributed bilaterally along the intraparietal sulci and in the precuneus, as well as in the left middle temporal gyrus and posterior cingulate. Our results support the assumption of a central semantic representation of numerical quantity that relies on a common parietal network shared among notations. 2001 Academic Press

Key Words: parietal cortex; distance effect; notation effect; numerical comparison.

\section{INTRODUCTION}

How do we go from seeing a word to accessing its meaning? Classical models of word processing postulate that words are initially recognized in modalityspecific input lexicons before contacting a common semantic representation (Caramazza, 1996; Morton, 1979). This predicts that areas which are engaged in

\footnotetext{
${ }^{1}$ To whom correspondence should be addressed at the Unité INSERM 334, Service Hospitalier Frédéric J oliot, CEA, 4 Place du Général Lederc, 91401 Orsay Cedex, France. Fax: +33169867816. E-mail: dehaene@shfj.cea.fr.
}

semantic-level processing should activate in direct correlation with the amount of semantic manipulation required by the task and do so independent of the modality of presentation of the concept (Chao et al., 1999; Gorno-Tempini et al., 1998; Le Clec'H et al., 2000; Perani et al., 1999; Vandenberghe et al., 1996).

Here, we attempt to identify the cerebral areas engaged in the coding and internal manipulation of an abstract semantic content, the meaning of number words. Although numbers can be written in multiple notations, such as words or digits, the parietal lobes are thought to comprise a notation-independent representation of their semantic content as quantities. According to the "triple-code model" of number processing (Dehaene and Cohen, 1995), the left and right ventral occipitotemporal areas are initially involved in identifying the visual shapes of words and Arabic numerals. Word identification is thought to be strictly left-lateralized and to rely on the left "visual word form area," a region of the left fusiform gyrus which is involved in the invariant recognition of visual words (Shallice, 1988). The identification of Arabic numerals, by contrast, is predicted to implicate the left fusiform, but also its right-hemispheric homolog. In the triple-code model, both of these areas send information about the identified word or digit to the bilateral parietal lobes, where numerical quantity is represented and manipulated.

The amount of engagement of the quantity system can be manipulated parametrically by engaging subjects in a number comparison task. When deciding which of two numbers is the larger, subjects are systematically slower and make more errors as the distance between the numbers decreases. This "distance effect" is called "semantic" because performance depends only on numerical proximity, an abstract parameter which is unrelated to the shapes of digits or number words. Subjects appear to quickly convert those arbitrary visual shapes into an amodal representation of the corresponding quantity and its numerical proximity to other numbers. Convergence to the same quantity code, independent of the input notation, is 
supported by the empirical observation that the semantic distance effect has the same size and shape whether numbers are presented as Arabic digits, written words, or even dot patterns (Buckley and Gillman, 1974; F oltz et al., 1984; Tzeng and Wang, 1983).

We used functional magnetic resonance imaging (fMRI) and event-related potentials (ERPS) to identify the cerebral correlates of the semantic distance effect. Brain activity was imaged in a parametric event-related design while subjects decided whether a 2-digit number was smaller or larger than 65 . We relied on the additive-factors method (Dehaene, 1996; Sternberg, 1969) and varied orthogonally two parameters: numerical distance (close, intermediate, or far from 65) and notation (Arabic or verbal, i.e., written number words). This design allowed us to search for areas whose activation level correlated with numerical distance, independent of number notation. The triple-code model predicted that neural correlates of this distance effect would be observed in the left and right parietal lobes. We also examined the differences in activation between the two number notations, in order to test the model's prediction of a common activation in the left fusiform gyrus when identifying words and Arabic numerals and of a greater activation of the right fusiform gyrus when identifying Arabic numerals. Finally, by timing the onset of those notation and distance effects using ERPs, we clarified the temporal organization of those activations.

\section{MATERIALS AND METHODS}

\section{Subjects}

Twenty-six healthy volunteers (16 men and 10 women; mean age 25 years) participated in the study ( 9 participants in the first fMRI protocol, 4 in the additional fast event-related fMRI experiment, and 13 in the ERP protocol) after giving their written informed consent. All subjects were right-handed according to the Edinburg inventory. The research project was approved by the regional ethical committee for biomedical research.

\section{Procedure}

In all experiments, subjects had to perform a numerical comparison task. They decided if a visually presented number was larger or smaller than a fixed memorized reference number (65) by pressing a button with their right or left hand according to instructions. This replicated an earlier psychological experiment (Dehaene et al., 1990), in which the choice of the reference 65 was shown to lead to a smooth distance effect in response times. Although it might be thought that numbers starting with the same decades digit as the reference are compared in a qualitatively different manner, owing to the need to focus on the unit digits, response time measurements disconfirmed this hypothesis by showing no discontinuities at the decade boundaries surrounding the reference number (Dehaene et al., 1990). Thus, perceptual or phonological similarity between the numbers appears to play a minor role relative to the influence of the continuous distance between the compared quantities. Based on those prior results, a list of 120 target numerals was constructed. In order to compensate for the nonlinear shape of the behavioral distance effect, which becomes progressively steeper as the target numbers get closer to the reference number, the targets were distributed in three categories of distances: numbers "close" to 65 (intervals 60-64 and 66-69), numbers moderately distant from 65 ("medium" distance, intervals 50-59 and 70-79), and numbers "far" from 65 (intervals 30- 49 and 80-99). Targets were sampled equally often from each of those three categories.

In different blocks, target numbers could be presented in two notations, Arabic or verbal numerals. Each trial began by the short presentation ( $200 \mathrm{~ms})$ of a white rectangle to draw subjects' attention, followed 2 s later by a number that was visible for 200 ms. Because of their small number of characters, Arabic numbers were presented in a larger font than verbal ones (Arabic $1.6 \times 1^{\circ}$ of visual angle; average of verbal stimuli, $\left.7.8 \times 0.8^{\circ}\right)$. Following each number, subjects responded as quickly as possible and their response was timed to the nearest millisecond. Trials were separated by a 14-s interstimulus interval (ISI) for the fMRI experiment and by a 3-s ISI for the ERP experiment. They were separated into four blocks, corresponding to all combinations of notation and response instruction, within which numerical distances were randomly intermixed. FMRI blocks comprised 30 trials each, while ERP blocks comprised 128 trials. Before each run, subjects practiced for a short period of 20 trials (3-s ISI).

\section{FMRI Acquisition and Analysis}

FMRI images were acquired following an event-related design. Stimulus onset was synchronized with the acquisition of the first slice in a series of seven volumes of 18 slices each (one volume every $2 \mathrm{~s}$ ). We used a gradient-echo echo-planar imaging sequence sensitive to brain oxygen-level dependent (BOLD) contrast (18 contiguous axial slices, $6 \mathrm{~mm}$ thickness, TR/ TE $=2000 / 40 \mathrm{~ms}$, in-plane resolution $3 \times 4 \mathrm{~mm}^{2}, 64 \times$ 64 matrix) on a 3-T whole-body system (Bruker, Germany). High-resolution anatomical images (3D gradient-echo inversion-recovery sequence, $\mathrm{TI}=700 \mathrm{~ms}$, $\mathrm{TR}=1600 \mathrm{~ms}$, FOV $192 \times 256 \mathrm{~mm}^{2}$, matrix $128 \times$ $128 \times 256$, slice thickness $1.5 \mathrm{~mm}$ ) were al so acquired.

FMRI analyses were performed using statistical parameter mapping software (SPM 99). I mages were cor- 
rected for subject motion and realigned using sinc interpolation. Anatomical images of each subject were normalized to Talairach space using the template of the Montreal Neurological Institute. Functional images were then normalized using the same transformation and smoothed (Gaussian filter width $=15 \mathrm{~mm}$ ) for group analysis. We limited our SPM analysis to the impact of numerical distance and notation factors. For each subject, correct trials were averaged together according to the $3 \times 2 \times 7$ combinations of these factors and time. All averaged trials from nine subjects were then analyzed together using the generalized linear model of SPM 99 to model each trial type by the SPM 99 standard hemodynamic function and its derivative. The data were high-pass filtered using a set of discrete cosine basis functions (temporal cut-off period of $120 \mathrm{~s}$ ) and low-pass filtered using a Gaussian filter (4 s). Unless otherwise stated, contrasts were examined with a voxel-wise significance level of 0.05 corrected for multiple comparisons across the brain volume. An $\mathrm{F}$ test was used to identify regions showing any activation difference between the three distance categories. This $F$ test was masked exclusively by an $F$ test for the notation $\times$ distance interaction $(P=0.05)$ to report only distance effects that were invariant across notations. We also examined a t test for the main effect of notation. Finally, we examined the interaction between distance and notation with an $F$ test. All contrasts were masked $(P=0.05)$ inclusively by the overall $t$ test for a positive BOLD effect to focus only on activations relative to the intertrial resting period.

For each observed cluster, we then applied a classical random-effect analysis of variance (ANOVA) on the peak activation at the most significant voxel to test distance $\times$ time, notation $\times$ time, and distance $\times$ notation $\times$ time interactions without any a priori hypothesis on the shape of the hemodynamic response, as previously described (Pinel et al., 1999) (threshold of $P<0.05$ corrected with the Greenhouse-Geisser method). In an additional ANOVA not reported here, we also verified that all the regions reported here were unaffected by the side of the motor response (left or right) nor by the numerical size of the stimuli (larger or smaller than 65).

\section{ERP Acquisition and Analysis}

The electroencephalogram was recorded from a 128electrode geodesic sensor net with a reference on the vertex and sampled at $125 \mathrm{~Hz}$. Trials with incorrect responses, voltages exceeding $\pm 100 \mu \mathrm{V}$, transients exceeding $\pm 50 \mu \mathrm{V}$, electro-oculogram activity exceeding $\pm 70 \mu \mathrm{V}$, or response times outside a 250-1500 ms interval were rejected. Stimulus-locked ERPs were computed separately for each combination of notation and distance by averaging the remaining trials in synchrony with the stimulus onset, while response-locked
ERPs were obtained by averaging in synchrony with the key press. ERPs were band-pass filtered (0.5-20 $\mathrm{Hz}$ ) and corrected for baseline over a 200-ms window prior to stimulus onset.

The stimulus-locked ERP analysis addressed early cognitive events. We examined notation effects by contrasting the Arabic and verbal conditions, as well as the first appearance of a semantic effect as indicated by a significant influence of distance within each notation. The response-locked ERP analysis was used to identify a common stage affected only by distance, by going backward in time starting 140 ms prior to the key press, which is the estimated time when response programming occurs (Dehaene, 1996). Experimental conditions were compared sample by sample with nonparametric Kolmogorov-Smirnov tests. A difference was considered significant when it appeared for five consecutive samples at $\mathrm{P}<0.05$ simultaneously on eight electrodes. We then selected groups of adjacent electrodes and time windows at which the considered effect reached the highest level of significance. Electrodes located symmetrically in the opposite hemisphere were also analyzed. The sel ected electrodes and time windows were the following: $\mathrm{t}=120-152 \mathrm{~ms}$, medial occipital area (electrodes 67, 72, 73, 77, 78); $\mathrm{t}=$ 168-200 ms, left parietotemporal region (electrodes 57, $58,64,65$ ) and right homologous region (electrodes 91, $96,97,101)$; and $t=352-288 \mathrm{~ms}$ prior to the response, left parietal region (electrodes 58, 59, 64, 65), right homologous region (electrodes 91, 92, 96, 97), and medial posterior occipital area (electrodes 76, 75, 83, 82). Voltages averaged over those electrodes and time windows were then entered into an ANOVA to test for effects of notation, distance, notation $\times$ distance interaction, and interaction of those tests with hemisphere, with a criterion of $\mathrm{P}<0.05$.

Scalp topographies were visualized using 2D maps constructed by spherical spline interpolation (Perrin et al., 1989) and realistic 3D maps taking into account the actual distribution of electrodes on the head. Dipole models were generated with BESA99 for Windows (Scherg and Berg, 1990) using a spherical four-shell approximation of the head. To constrain the models, dipoles were placed at locations in Talairach space where the most significant fMRI effects were observed for notation or distance effect. The program then selected the dipole orientation and strength to match the scalp topography of the ERP difference at the time point of interest. The amount of variance thus explained by constrained models approximately reflects the contribution of brain areas identified with fMRI to the corresponding ERP topography.

\section{Additional Fast Event-Related FMRI Experiment}

Four additional subjects participated in a fast eventrelated fMRI experiment (same as above, except EPI 


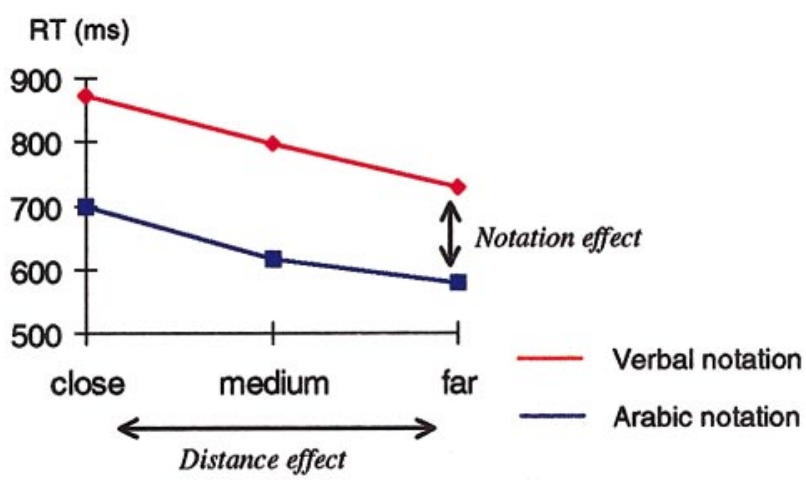

FIG. 1. Averaged response times of all subjects as a function of numerical distance and number notation. Data are reported in red for verbal stimuli and in blue for Arabic digits. The monotonic decrease of response time with distance reflects the distance effect for both notations, while the constant gap between the red and the blue curves represents a notation effect independent of the numerical content of the stimuli.

imaging with TR $=2400 \mathrm{~ms}, 26$ axial slices, $4 \mathrm{~mm}$ thickness, in-plane resolution $3.75 \times 3.75 \mathrm{~mm}^{2}$ ). Task and experimental conditions were the same as in the group protocol. In each of four blocks, all Arabic numbers in the range 30-99, plus 10 blank trials without any target, were presented twice in random order, with a 2.4-s intertrial interval. Images were processed as previously described. Results were analyzed on an individual-by-individual basis. We created with SPM99a model with two functions modeling either a constant activation on each trial or an activation whose intensity was modulated linearly by numerical distance, plus their time derivatives. A simple t test for the contribution of the distance-modulated predictor $(\mathrm{P}=$ 0.001 uncorrected) was then used to identify cerebral correlates of the semantic distance effect.

\section{RESULTS}

\section{Behavioral Data}

An ANOVA on median correct response times, with notation and distance as within-subjects factors and group (fMRI or ERP subjects) as a between-subjects factor, revealed significant effects of all three factors. First, verbal numerals were compared 169 ms slower than Arabic numerals $(\mathrm{F}(1,20)=64.8, \mathrm{P}<0.0001)$. Second, response times decreased monotonically with numerical distance from 65 (respectively 786, 708, and 654 ms for close, medium, and far categories; $F(2,40)=$
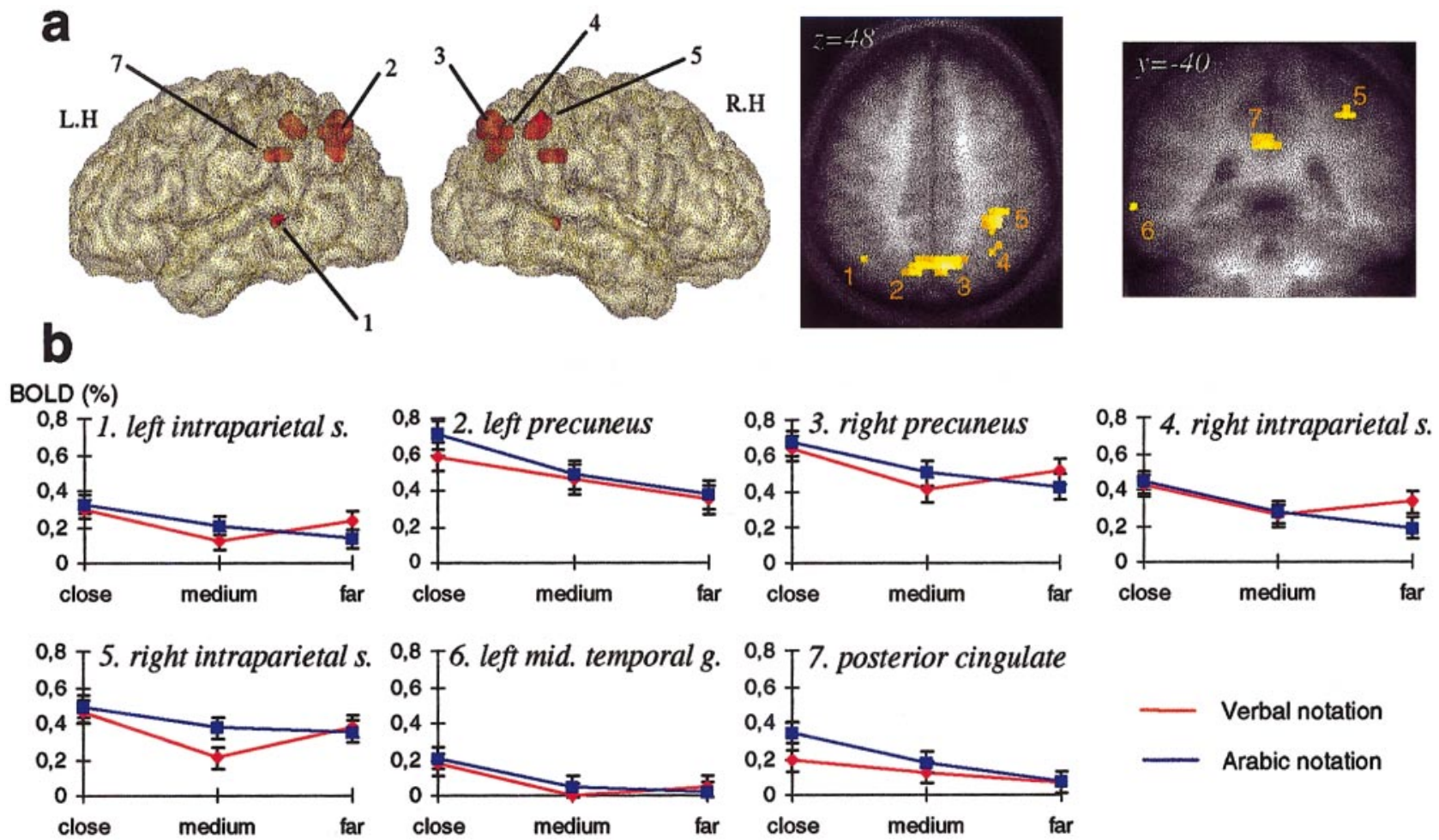

FIG. 2. $f M R I$ of the distance effect in the group analysis. (a) Localization of brain areas affected by numerical distance (P $<0.05$ corrected). On the left, 3D representation of these regions on a transparent brain ( $\mathrm{L} . \mathrm{H}$, left hemisphere; R.H, right hemisphere). On the right, detailed position of each region on an axial and a coronal anatomical slice. (b) For each identified region, plots show percentage activation for the most significant voxel as a function of numerical distance in the verbal (red) and Arabic (blue) conditions. Length of statistical significance bars represents $1 / 2 \mathrm{~V}(\mathrm{MSE} / \mathrm{n})$, where MSE is the mean square error associated with the subject $\times$ conditions interaction and $n$ the number of subjects. 

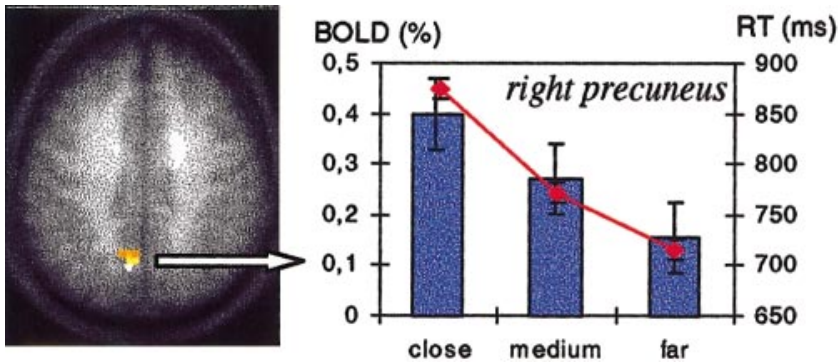

FIG. 3. Brain area presenting a strictly monotonic decrease of percentage activation (blue columns) with numerical distance ( $\mathrm{P}<$ 0.05 corrected) similar to the pattern of reaction times (red curve). Data are averaged across notations.

78.0, $\mathrm{P}<0.0001)$. Third, subjects in the fMRI group were $122 \mathrm{~ms}$ slower than subjects in the ERP group $(\mathrm{F}(1,20)=5.0, \mathrm{P}=0.037)$, presumably due to the slower pace of trials. Crucially, none of the interactions reached significance, and in particular there was no interaction of the notation and distance factors $(F(2,40)=3.20, P>0.05 ; \mathrm{Fig} .1)$. The finding of $\mathrm{a}$ similar distance effect in both notations supports a serial model of the task, with two successive stages of notation-specific identification and access to quantity, and it validates our search for distinct cerebral substrates of those stages.

\section{Neural Basis of the Semantic Distance Effect}

Numerical distance had a significant effect on fMRI activations in a parietal circuit comprising a right in- traparietal focus, bilateral posterior intraparietal foci, and a bilateral activation of the precuneus (Fig. 2a; Table 1). Marginally significant effects of distance were also found in the left middle temporal gyrus and the posterior cingulate cortex. Several observations confirmed that number notation was irrelevant to those areas. First, activation was not affected by a main effect of number notation, nor did the numerical distance effect interact with notation (Table 1). Second, the distance effect was found to be significant within each type of notation. In all areas, whether numbers were presented in Arabic or in verbal notation, smaller numerical distances were associated with higher activation levels (Fig. 2b).

The shape of the fMRI responses to close, medium, and far numbers was somewhat variable, however. We ther efore searched for areas which presented a pattern of activation directly analogous to the behavioral data, namely a monotonic variation of activation with numerical distance. When masking the main effect of distance by four contrasts for close $>$ medium and medium $>$ far in each notation, only the left precuneus remained (coordinates $-8,-68,44$; Fig. 3 ).

We also searched the whole brain for the presence of areas showing a distance by notation interaction. The SPM-based $\mathrm{F}$ test for this interaction revealed no cluster at a corrected $\mathrm{P}<0.05$ but did at a lower threshold (uncorrected $\mathrm{P}<0.001$ ). U sing thet test (see Materials and Methods), we identified voxels showing a greater distance effect for verbal numerals in superior parietal
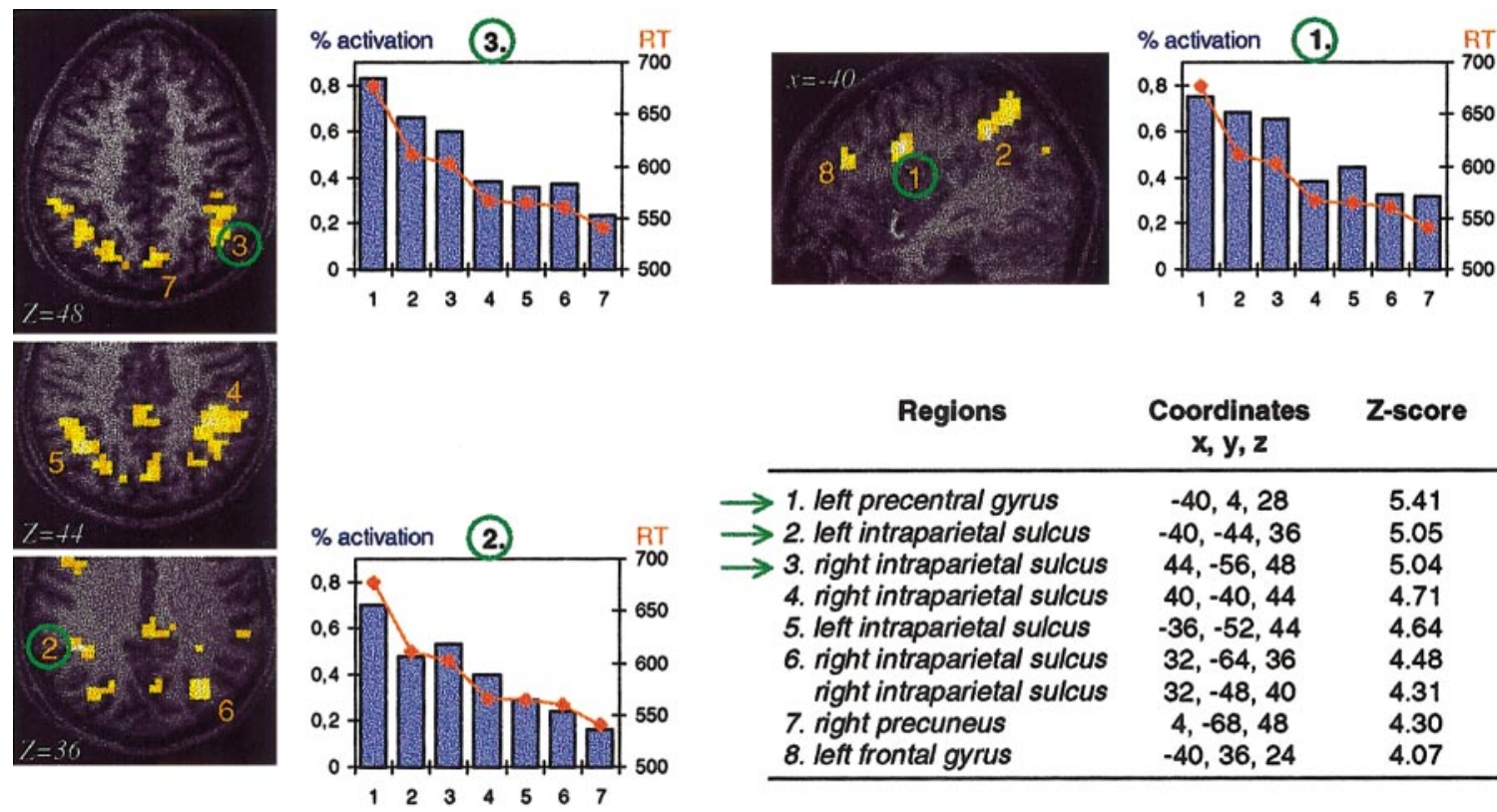

\begin{tabular}{lccc}
\multicolumn{1}{c}{ Regions } & $\begin{array}{c}\text { Coordinates } \\
\mathbf{x}, \mathbf{y}, \mathbf{z}\end{array}$ & Z-score & $\boldsymbol{P}_{\text {uncorrected }}$ \\
\hline 1. left precentral gyrus & $-40,4,28$ & 5.41 & $<0.001$ \\
$\rightarrow$ 2. left intraparietal sulcus & $-40,-44,36$ & 5.05 & $<0.001$ \\
$\rightarrow$ 3. right intraparietal sulcus & $44,-56,48$ & 5.04 & $<0.001$ \\
4. right intraparietal sulcus & $40,-40,44$ & 4.71 & $<0.001$ \\
5. left intraparietal sulcus & $-36,-52,44$ & 4.64 & $<0.001$ \\
6. right intraparietal sulcus & $32,-64,36$ & 4.48 & $<0.001$ \\
$\quad$ right intraparietal sulcus & $32,-48,40$ & 4.31 & $<0.001$ \\
7. right precuneus & $4,-68,48$ & 4.30 & $<0.001$ \\
8. left frontal gyrus & $-40,36,24$ & 4.07 & $<0.001$ \\
\hline
\end{tabular}

FIG. 4. Distance effect in the single-subject analysis. Axial and sagittal slices show the main brain areas where activation was correlated with numerical distance ( $P<0.001$, uncorrected). Green circles indicate the three most significant regions (corrected $P<0.05$ ); for each of them, graphs show averaged percentage activation (blue columns) and reaction time (red curve) as a function of numerical distance, categorized into seven levels of numerical distance from 65 [distances 1 (60- 64 and 66-70), 2 (55-59 and 71-75), 3 (50-54 and 76-80), 4 (45- 49 and 81- 85), 5 (40-44 and 86-90), 6 (35- 39 and 91-95), and 7 (30-34 and 96-99)]. Region numbers refer to the table, where Talairach coordinates of the most significant maxima as well as $\mathrm{Z}$ scores and voxel $\mathrm{P}$ values are reported. 
TABLE 1

Talairach Coordinates of Foci Showing Significant Notation and Distance Effects in the SPM Analysis

\begin{tabular}{|c|c|c|c|c|c|c|}
\hline \multirow[b]{2}{*}{ Region } & \multirow[b]{2}{*}{$\begin{array}{c}\text { Coordinates } \\
(x, y, z)\end{array}$} & \multicolumn{2}{|c|}{ SPM analysis } & \multicolumn{3}{|c|}{ ANOVA analysis } \\
\hline & & $\begin{array}{l}T \text { or } F \\
\text { value }\end{array}$ & $\mathrm{P}_{\text {corrected }}$ & $\begin{array}{c}\text { P notation } \\
\text { effect }\end{array}$ & $\begin{array}{l}\text { P distance } \\
\text { effect }\end{array}$ & $\mathrm{P}$ interaction \\
\hline
\end{tabular}

Verbal > Arabic notation Right occipital lobe Left occipital lobe Left precentral sulcus

Arabic $>$ verbal notation Right fusiform gyrus

Right middle temporal gyrus

Right inferior parietal lobule

Left inferior parietal lobule

Right middle frontal gyrus

Left middle frontal gyrus

Distance effect

Right intraparietal sulcus

Right intraparietal sulcus

Right precuneus

Left precuneus

Left parietal

Left middle temporal gyrus

Posterior cingulate

$\begin{array}{lc} & \mathrm{T} \\ \text { 16, -80, 0 } & 4.63 \\ -24,-88,4 & 4.61 \\ -52,8,32 & 8.56 \\ & \mathrm{~T} \\ 44,-64,-8 & 4.67 \\ 60,-16,-8 & 5.37 \\ 56,-44,40 & 10.80 \\ -64,-40,40 & 6.97 \\ 24,40,40 & 7.00 \\ -36,36,44 & 8.29 \\ & \mathrm{~F} \\ 40,-44,48 & 18.62 \\ 36,-64,48 & 13.94 \\ 8,-72,52 & 21.68 \\ -4,-72,44 & 17.84 \\ -40,-68,48 & 14.60 \\ -72,-40,0 & 15.57 \\ 0,-40,32 & 16.82\end{array}$

4.63

4.61

8.56

4.67

5.37

10.80

6.97

7.00

$\mathrm{F}$

8.62

21.68

17.84

14.60

16.82

0.022
0.024
$<0.001$

0.019
0.001
$<0.001$
$<0.001$
$<0.001$
$<0.001$

$<0.001$
0.019
$<0.001$
0.001
0.011
0.005
0.002

0.005
0.003
0.001
0.048
-
0.018
0.011
0.032
0.008
-
-
-
-
-
-

effect

$\mathrm{P}$ interaction

Note T values and corrected voxel-based P values are shown for each of the statistical tests in the left column. Results of a conventional analysis of variance with subjects as the random factors appear at right. Only $\mathrm{P}$ values below 0.10 are reported.

lobe (coordinates $-16,-44,76$ and $8,-48,72$ ) and voxels showing a greater distance effect for Arabic numerals in left posterior parietal cortex $(-8,-60,52)$ and frontal sites: bilateral superior frontal gyrus $(-32$, 4,48 and 16, 16, 64), left precentral gyrus $(-60,8,44)$, left cingulate $(-16,32,20)$, left basal ganglia $(-4,24$, $0)$, and right medial frontal gyrus $(20,44,-4)$. Those effects were small, however, and might have been due to only a small number of subjects since none of them survived a classical analysis of variance with subjects as the random factor $(P>0.05)$.

\section{Parallel Shapes of the Distance Effect in Imaging and Behavior}

The main fMRI experiment analyzed the distance effect using only three discrete distance levels: close, medium, or far numbers. In four additional subjects, we examined whether parietal activation varies continuously in parallel to numerical distance at the single-subject level. In this experiment, which used fast event-related fMRI, the subjects saw 2-digit Arabic numerals presented at a rate of one number every $2.4 \mathrm{~s}$, thus permitting a fivefold increase in the number of trials and a homogeneous sampling of the numerical interval 31-99 (see Materials and Methods). Figure 4 shows the results obtained in one subject. The regions whose activation was modulated by numerical distance were located in the left and right intraparietal sulci, the precuneus, and the left precentral gyrus. In each of those regions, activation decreased quasi-monotonically as the numerical distance increased, in tight parallel with the subject's response time curve (Fig. 4). As previously described (Dehaene et al., 1990; Hinrichs et al., 1981), response times were a convex-upward function of numerical distance and were better predicted by a logarithmic regression $\left(r^{2}=96.5 \%\right)$ than by a linear regression with distance $\left(r^{2}=84.8 \%\right)$. Similarly, in six of the seven foci at which a distance effect was found, activation was better predicted by the logarithm of distance than by distance itself (mean $r^{2}=89.0 \%$ with $\log$ D versus $81.3 \%$ for lin D). Similar observations of a strong correlation between intraparietal activation and the behavioral distance effect were also obtained in two other subjects. Only one of them, however, showed an additional correlation with the left and right precentral cortex. A fourth subject failed to show any strong correlation between brain activation and numerical distance or indeed any strong activation relative to the "null event," whose frequency (oneeighth of trials) might have been slightly too low for this type of fast event-related paradigm.

\section{Neural Basis of Notation-Specific Effects}

The cerebral areas showing more activity for Arabic than for spelled-out numerals were the right fusiform gyrus, the right middle temporal gyrus, and a large set of bilateral frontoparietal regions (Fig. 5; Table 1) distinct from those showing a distance effect (Fig. 6). 
Conversely, greater activity to spelled-out numerals was observed in bilateral extrastriate occipital areas as well as the left precentral gyrus (Fig. 5; Table 1). Because of an earlier report of greater activity to letter strings than to digit strings in the left inferior temporal region (Polk and Farah, 1998), we searched this region at a lower threshold ( $P=0.001$, uncorrected). This analysis indeed revealed a small cluster in the left ventral anterior fusiform region (coordinates -52 , $-48,-8 ; t=4.38$, corrected $P=0.055)$. This region falls anterior to the published coordinates of the visual word form area (Cohen et al., 2000a), where a strong activation was also observed (maximum at $-52,-60$, $-4)$, but where the activation was identically intense for verbal stimuli $(t=16.4$ ) and for Arabic stimuli ( $t=$ 14.1; t test for difference $=1.71$, n.s.).

In most of those areas, activation was affected only by notation and showed no effect of semantic distance nor any distance by notation interaction (Table 1). The sole exception was the right middle temporal gyrus, where a trend toward an interaction of notation and distance was found $(P=0.054)$. In this region, which was approximately symmetrical to the left middletemporal region where a main effect of distance was found, activation decreased monotonically with numerical distance, but only for Arabic stimuli (ANOVA restricted to Arabic stimuli, $\mathrm{F}(12,96)=2.43, \mathrm{P}=0.07)$, not for verbal stimuli (ANOVA restricted to verbal stimuli, $F(12,96)=1.42, P=0.245)$.

\section{Temporal Decomposition U sing Event-R elated Potentials}

According to the proposed model of number comparison, following a notation-specific stage of stimulus identification, processing converges toward a common process of quantity comparison. I dentification is slower for verbal than for Arabic notation, as evidenced by a main effect of notation on response times. This predicts that, in all regions affected by the distance effect, the neural response should be delayed for verbal numerals relative to Arabic numerals. We tested this by using the high temporal resolution afforded by event-related potentials.

The early components of the evoked response were affected only by number notation. A first effect of notation was identified 120 ms after stimulus onset, with a peak difference at $136 \mathrm{~ms}(\mathrm{~F}(1,12)=13.93, \mathrm{P}=$ $0.003)$. It was characterized by a greater occipital P1 for Arabic digits than for verbal stimuli, reflecting the physical differences between 2-digit numerals and letter strings. A dipole model indicated that its topography fit well with the greater occipital activation to verbal numerals observed in fMRI (Fig. 7); two symmetrical occipital dipoles (Talairach coordinates 16, $-64,7$ and $-16,-64,7)$ accounted for $96.2 \%$ of variance. At this stage, no semantic effect was observed (distance $F(2,24)<1$; notation $\times$ distance $F(2,24)<1$ ).
About 40 ms later, a second notation effect, peaking at $184 \mathrm{~ms}$, appeared on lateral occipitotemporal sites $(F(1,12)=12.95, P=0.004)$ with a right lateralization (hemisphere $\times$ notation $F(1,12)=11.61, P=0.005)$. This effect coincided with the peak of the N1 negativity, which was smaller and left-lateralized for verbal stimuli and larger and bilateral for Arabic stimuli (Fig. 7). A model with two symmetrical dipoles characterized this topography as arising from a greater right ventral visual activation $(48,-56,-8)$ to Arabic numerals, in agreement with the $\mathrm{fMRI}$ difference observed in this region. Such a model explained $76.4 \%$ of variance.

The first trace of a distance effect was observed on the same time window. A triple interaction of notation, distance, and hemisphere $(\mathrm{F}(2,24)=3.52, \mathrm{P}=0.045)$ indicated that distance had a significant influence on right temporal voltages for numbers in Arabic notation $(\mathrm{F}(2,24)=22.11, \mathrm{P}<0.001)$, but not for verbal numbers $(F(2,24)<1)$. This indicates that, as predicted, there is a time period in which the semantic content of Arabic numerals is al ready being processed while verbal numerals are lagging behind. No semantic effect appeared with verbal numerals until $220 \mathrm{~ms}$ (peak at $232 \mathrm{~ms}, \mathrm{~F}(2,24)=4.99, \mathrm{P}=0.015)$, or about 60 ms later than with Arabic stimuli. This time lag confirms that at least part of the 169-ms delay for verbal numerals observed in behavioral performance is due to a slower access to quantity information in verbal than in Arabic notation.

To visualize the topography of the distance effect independent of any influence of notation, we removed notation-induced identification delays by computing response-locked ERPS. According to the proposed model of number comparison, all processing stages following stimulus identification unfold identically in both Arabic and verbal notation. Hence, by looking backward in time from the point of the response, one should find a time window at which there is a common topography of the distance effect for numbers in Arabic and in verbal notation. Indeed, a distance effect was identified starting about 350 ms before the response (peak difference around -320 ms) (Fig. 8). On bilateral occipitoparietal sites, voltage varied monotonically with semantic distance (left and right parietotemporal $F 2,24)=3.57, P=0.047$; occipital $F(2,24)=4.26, P=$ $0.029)$, and this effect did not interact with notation (parietotemporal $\mathrm{F}(2,24)<1$; occipital $\mathrm{F}(2,24)=2.50$, $P=0.107)$. Of the variance, $67.9 \%$ could be explained by two dipoles placed at bilateral parietal locations corresponding to the most important intraparietal activation of the fMRI distance effect $( \pm 40,-44,48)$. The fit was slightly improved adding two bilateral dipoles at locations analogous to the precuneus sites $( \pm 8,-72$, 52 ) observed in fMRI (74.1\% of variance accounted for). Dipole localizations directly proposed by BESA suggested, however, the existence of frontal sources. Indeed, a better fit was obtained adding a left precentral 
Arabic $>$ verbal notation

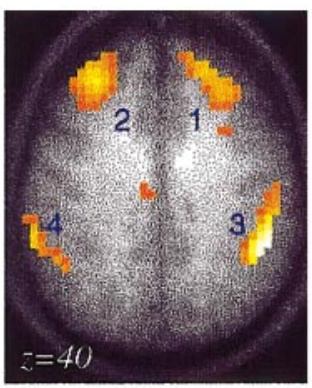

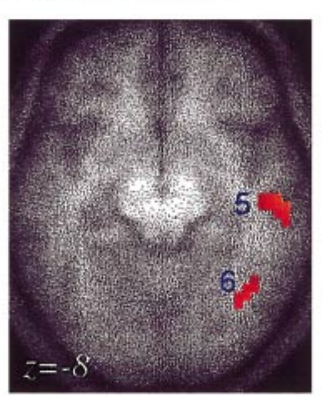

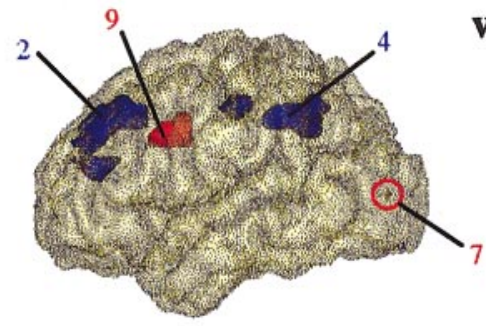

verbal > Arabic notation
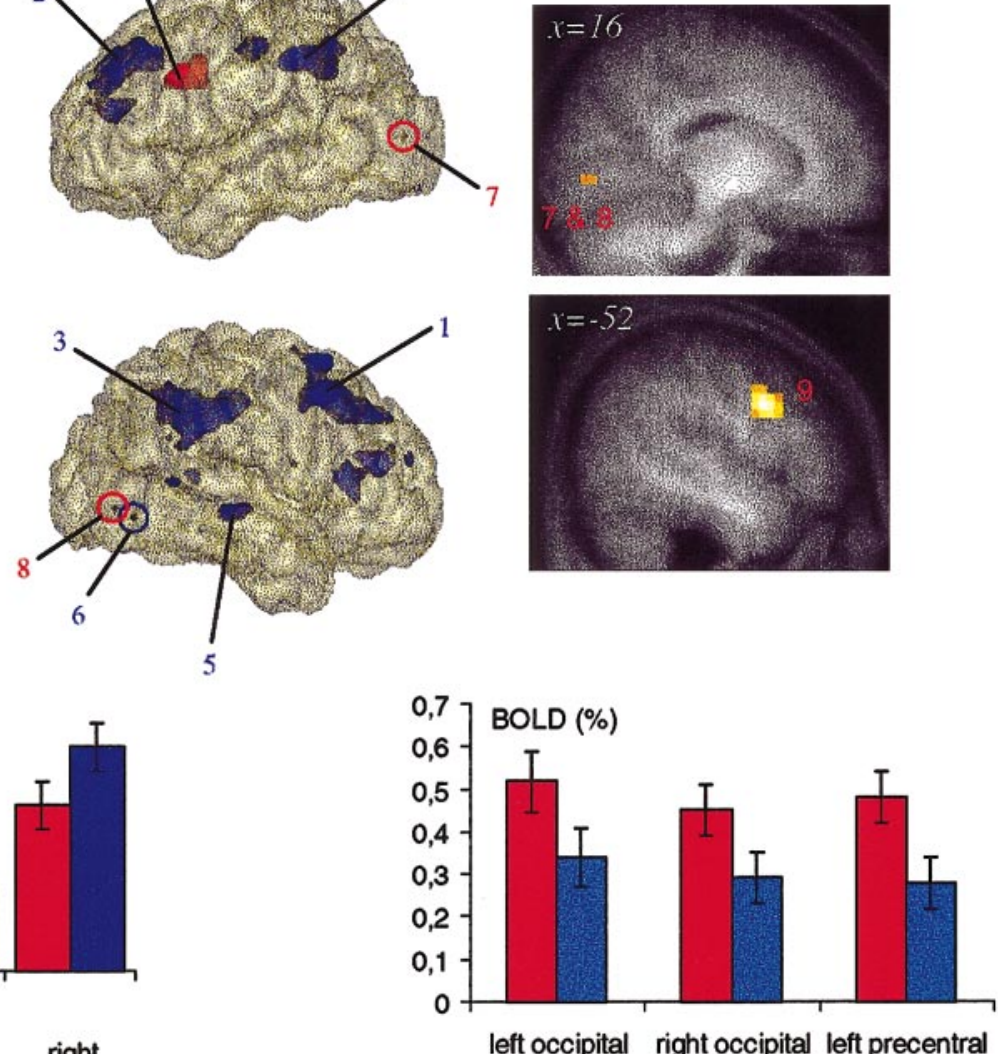

right mid. left mid. right inf. left inf. right mid. right prefrontal prefrontal parietal parietal Temporal fusiform

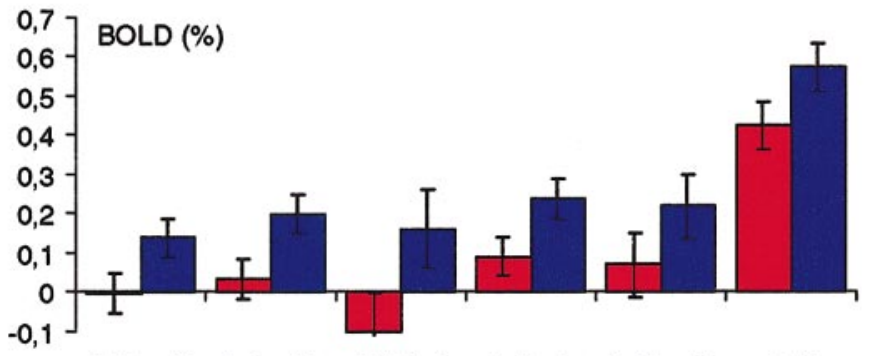

1 2 3 4 5 6

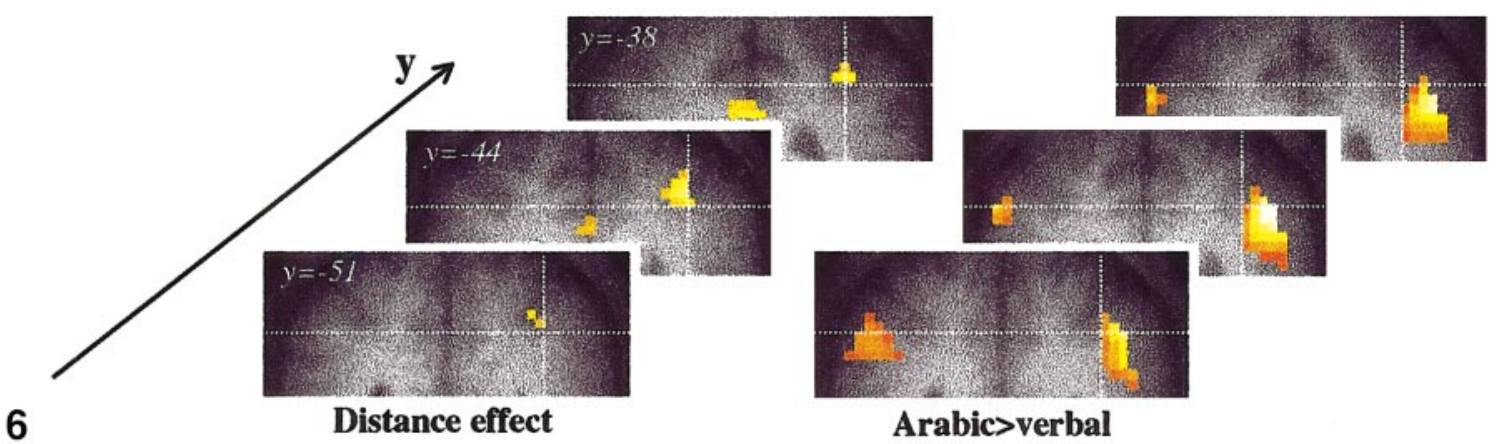

FIG. 5. Notation effects in the group analysis. Top row: Localization of brain areas with a significant notation effect (P $<0.05$ corrected); on the left, regions with stronger activation for Arabic than for verbal numerals are plotted on axial slices $(P=0.001$ for the right ventral slice for a better visualization); on the right, regions with stronger activation for verbal than for Arabic numerals are shown. In the middle, 3D representations of these regions (in blue for Arabic and in red for verbal) on a transparent brain. Because of the stringent statistical test (P $<0.05$ corrected), some ventral areas are reduced to a few voxels and are surrounded by a circle for a better visualization. Bottom row: Percentage activation of the most significant activated voxels in response to Arabic (blue columns) and to verbal (red columns) stimuli. Length of statistical significance bars represents $1 / 2 \sqrt{ }(M S E / n)$, where MSE is the mean square error associated with the subject $\times$ conditions interaction and $n$ the number of subjects.

FIG. 6. Respective locations of the distance effect and the notation effect in the right parietal lobe. Left: Voxels identified with the $F$ test on numerical distance $(P<0.05$, corrected). Right: Voxels identified with the Arabic $>$ verbal contrast $(P<0.05$, corrected). Dotted lines serve as a reference. Regions showing a distance effect occupy a superior and internal location in the depth of the intraparietal sulcus, while regions involved in Arabic encoding involve the inferior and lateral sectors of the angular and supramarginal gyri.

source to the two parietal dipole model $(89.2 \%$ of variance accounted for) on a site analogous to the one obtained in our single-subject fMRI experiment $(-40$,
$4,28)$ and close to those reported in other types of quantity manipulation (Chochon et al., 1999; Stanescu et al., 2000; Zago et al., 2001). 
a

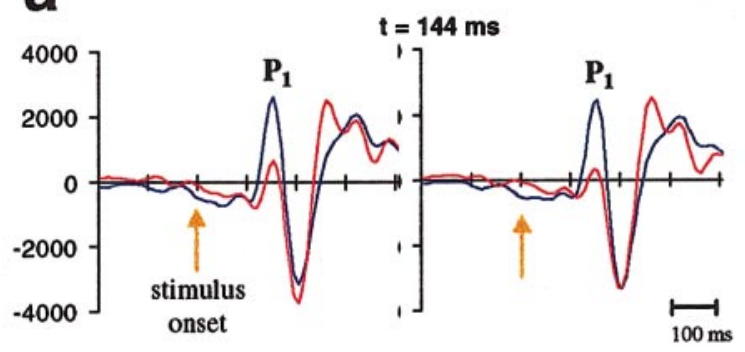

Arabic-verbal
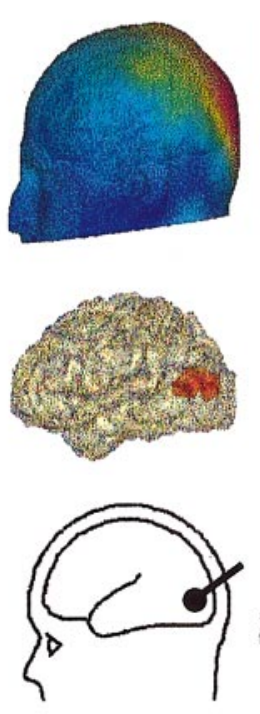

7
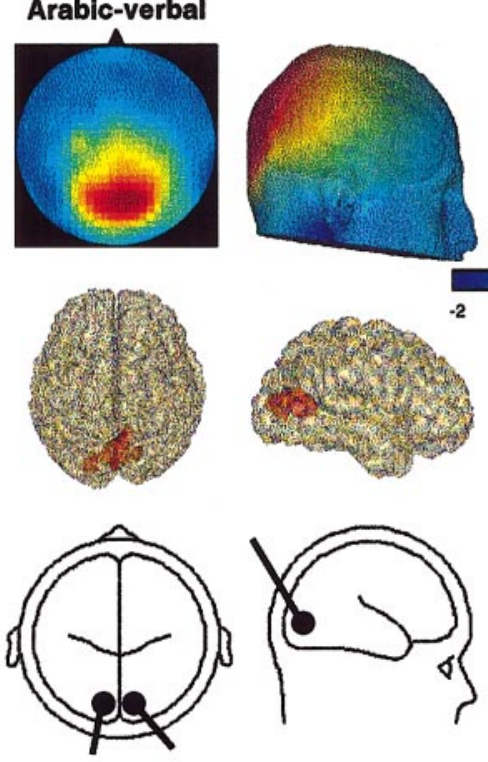

$\mathrm{t}=320 \mathrm{~ms}$

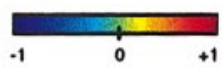

before response

Close-Far
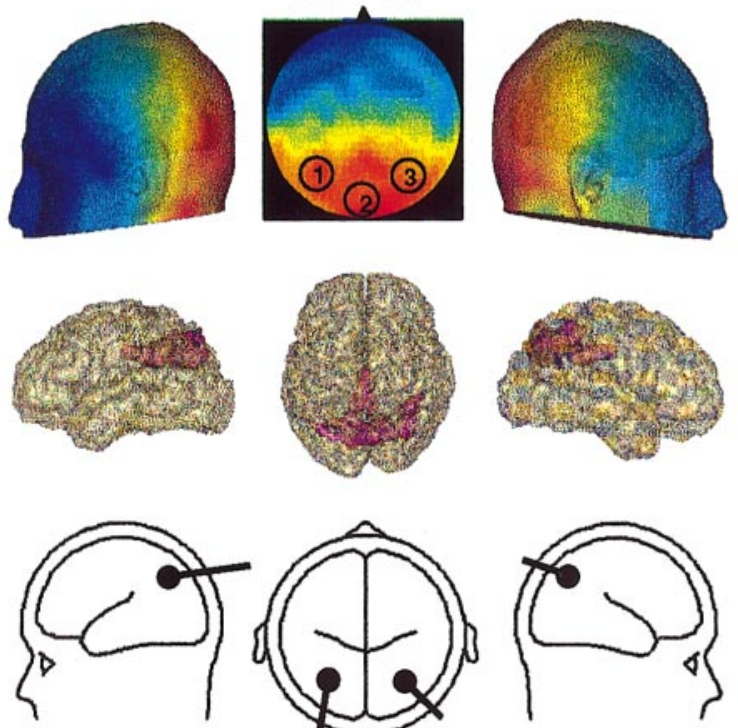

8
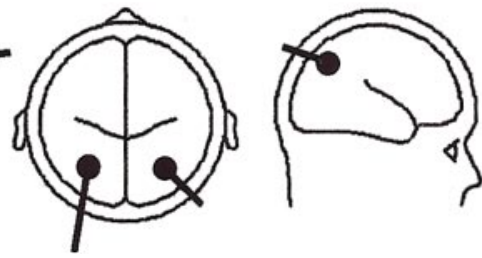

b

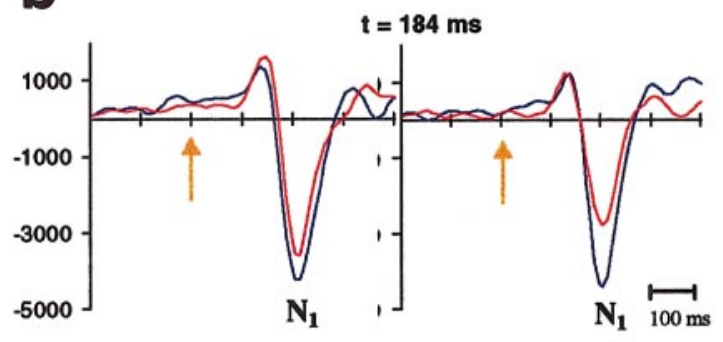

Arabic-verbal
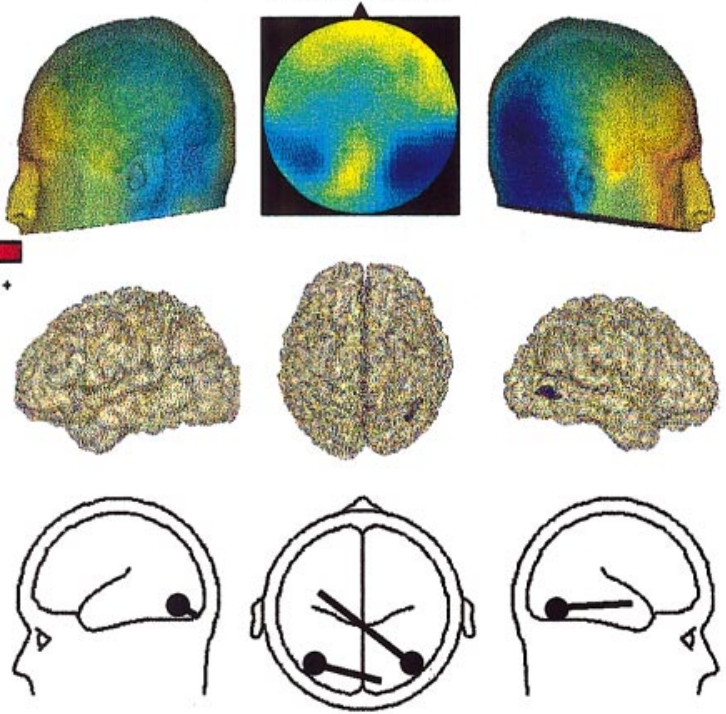

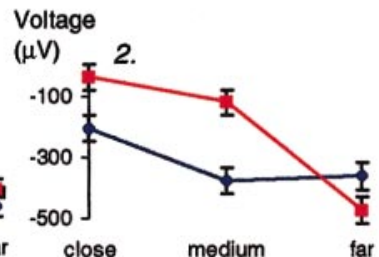

Voltage

$(\mu V) 0=3$.

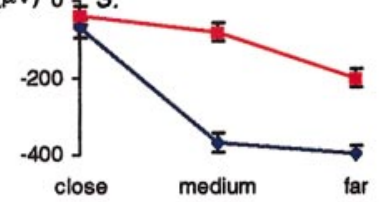

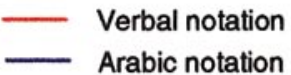

FIG. 7. ERP analysis of notation and distance effects. (a) First occipital notation effect on the P1, 144 ms after stimulus onset. Top row: Time curves of stimulus-locked ERP from occipital electrodes 72 and 77 plotted for verbal (red) and Arabic (blue) notations. Middle row: Topography of the difference between ERPs to Arabic and verbal stimuli, as seen in a 2D polar plot (at center) and a 3D plot. Bottom row: Possible anatomical correlates of this notation effect: occipital clusters showing greater activation to verbal than to Arabic stimuli in fMRI 


\section{DISCUSSION}

\section{ERPs, fMRI, and the Additive-Factors Method}

The present study relied on Sternberg's additivefactors method (Sternberg, 1969), which assumes that when two factors-here notation and distance- have additive effects on mean reaction time, they are likely to arise from processing stages that are separable and serially organized. Combining ERPs and fMRI with an additive-factors design provides a stringent test of a serial decomposition of the number comparison task in distinct stages of processing. FMRI can test the hypothesis of distinct stages by examining whether nonoverlapping sets of areas are affected by each factor. In the present case, no overlap was found between the areas influenced by notation and by distance, and only a single area showed a slight interaction of both factors. ERPs complemented fMRI, which is notoriously insensitive to fast serial processing, by showing that distinct temporal windows are affected by each factor. Finally, by testing the replicability of a semantic effect in different conditions-here the Arabic and verbal notations-the additive-factors method provided direct evidence for the existence of abstract semantic networks in the human brain that lie at the convergence of multiple notation-specific input systems (Chao et al., 1999; Gorno-Tempini et al., 1998; Le Clec'H et al., 2000; Perani et al., 1999; Vandenberghe et al., 1996).

\section{Semantic N umber Representation}

The semantic parameter of numerical distance was found to affect activation mostly in the bilateral parietal lobes, in the banks of the intraparietal sulcus, and in the precuneus, with small additional effects in the posterior cingulate cortex and left middle temporal region. Previous studies of number comparison and calculation processes have all found intense bilateral activation of the intraparietal sulcus relative to nonnumerical tasks (Chochon et al., 1999; Dehaene et al., 1999; Menon et al., 2000; Pesenti et al., 2000; Pinel et al., 1999; Stanescu-Cosson et al., 2000; Zago et al., 2001). The main contribution of the present work is to show that this parietal activation relates to a specific stage of the number comparison task. The amount of parietal activation is determined by a semantic param- eter, numerical distance, and this relation is invariant with the notation used to convey the numbers. This fits with the theoretical prediction that parietal cortices encode and manipulate numbers in a notation-independent quantity format (Dehaene and Cohen, 1995). Event-related potentials revealed that the distance effect starts about 200 ms following stimulus onset, with a differential delay depending on number notation and an eventual convergence to a common topography about 320 ms before the subject's response. Those values agree with previous ERP studies of single-digit number comparison in adults (Dehaene, 1996) and young children (Temple and Posner, 1998). They suggest that stimulus identification and conversion to the quantity format are nearing completion by about 200 ms.

As numerical distance decreases, response times and error rates increase, indicating that the task becomes more difficult. We doubt, however, that a general concept of task difficulty suffices to explain our results. Comparing verbal numerals was also slower and more error-prone than comparing Arabic numerals, and this effect was comparable in size to the semantic distance effect. Yet only distance, not notation, affected parietal activity. This evidence taken together with the results of a study of number approximation in which task difficulty was strictly controlled (Stanescu-Cosson et al ., 2000) suggests that parietal activity during tasks of semantic processing of numerical distance is not merely an artifact of the time and effort required by the task. The results are also unlikely to be contaminated by artifacts of eye, hand, or attentional movement because in all conditions subjects fixated on an identical 200-ms stimulus and made identical manual responses. Rather, the parietal lobe appears to make an important contribution to semantic numerical manipulations per se.

The issue of whether this contribution is or is not specific to the number domain remains open. The extent of the observed parietal areas suggests that most of them probably play a wider role in the mental manipulation of visuospatial information, of which comparison on the mental "number line" is merely a special case. Indeed, the precuneus and the dorsal intraparietal sulcus are active during many nonnumerical

and dipole model accounting for the ERP topography with two symmetrical occipital dipoles. (b) Same logic for the second notation effect appearing on the right temporal N1, $184 \mathrm{~ms}$ after stimulus onset plotted from left and right occipitotemporal electrodes 64 and 96 . F M RI showed a right fusiform cluster with greater activation to Arabic than to verbal stimuli. The topography was well described by two symmetrical ventral dipoles, with stronger activation in the right hemisphere.

FIG. 8. Main ERP effect of distance, seen at $320 \mathrm{~ms}$ before the motor response on responselocked ERP. On the left, 2D and $3 D$ views show the topography of the difference between cl ose numbers and far numbers. This topography is consistent with the parietal location of the fM RI clusters showing a distance effect and is well accounted for by two symmetrical parietal dipoles (lower left). On the right, for three el ectrode sites, graphs show voltage averaged in a time window surrounding the maximum effect, separately for verbal (red) and Arabic (blue) notations. At all sites, voltage decreases quasi-monotonically with numerical distance. Length of statistical significance bars represents $1 / 2 \sqrt{ }(\mathrm{MSE} / \mathrm{n})$, where MSE is the mean square error associated with the subject $\times$ conditions interaction and $n$ the number of subjects. 
visuospatial tasks, including eye movement (Kawashima et al., 1996), displacement of visual attention (Corbetta et al., 1998, 2000), spatial working memory (Diwadkar et al., 2000; Postle et al., 2000; Thomas et al., 1999), mental imagery (Mellet et al., 1996), mental rotation of three-dimensional objects or body parts (Carpenter et al., 1999; Kosslyn et al., 1998; Richter et al., 2000), and mental navigation on an internal map (Ghaem et al., 1997). The mental rotation task, in particular, bears some similarity with the present number comparison task in that both are thought to require internal manipulations of a nonsymbolic analogical representation on a continuum. Performance in mental rotation is determined by the angular distance between the two compared figures (Shepard and Metzler, 1971). Imaging studies comparable to ours have revealed a tight correlation between angular distance, behavioral performance, and the amount of activation of the superior parietal lobe (Richter et al., 2000; Harris et al., 2000; Tagaris et al., 1996).

Several of these visuospatial protocols have also reported a coactivation of the precuneus and the posterior cingulate, as observed in our study (Aguirre et al., 1996; Gron et al., 2000). Although the function of the posterior part of the cingulate cortex remains unclear, this brain area is anatomically connected to the medial part of the superior parietal lobe (Vogt and Pandya, 1987). Thus, the distance effect reported here in the posterior cingulate might reflect the involvement of a cinguloprecuneus circuit in magnitude comparison and more generally in visuospatial transformations.

Altogether, it seems likely that the ability to decide the location of a number relative to a fixed reference relies upon parietal circuitry whose wider functional role is the representation and manipulation of spatial information. This conclusion is consistent with psychological evidence for an automatic activation of spatial coordinates whenever numbers are processed (Dehaene et al., 1993), suggesting a mental representation of numbers in the form of a spatially extended "number line" (Dehaene, 1992; Gallistel and Gelman, 1992; Restle, 1970). Still, our study leaves open the possibility that a subset of parietal areas is specific to the numerical domain. The fact that parietal lesions can cause relatively selective impairments of number knowledge and calculation (Cipolotti et al., 1991) suggests some degree of domain specificity in parietal organization. Indeed, we note that, in the single-subject analysis derived from the fast event-related fMRI protocol (Fig. 4), the number-related activations fell in the inferolateral flank of the intraparietal sulcus, whereas spatial and attentional manipulations tend to produce more dorsal activations in the superior parietal lobe.

In order to resolve this issue, it would be useful to replicate the present findings in a singlesubject study, comparing the activated areas during number processing and during other nonnumerical spatial or attentional tasks. It would be particularly interesting to compare the neural correlates of the numerical distance effect with the nonsemantic distance effects that are also observed when judging, e.g., the relative locations, sizes, or luminances of two objects. Based on the above discussion, we tentatively predict that the posterior cingulate and precuneus would be systematically activated by various semantic and nonsemantic comparison tasks, while the lateral bank of the intraparietal sulcus might perhaps be more specifically associated with number processing.

The implication of the left precentral gyrus in quantity manipulation remains unclear; while we failed to find any semantic influence on this area in our fMRI group analysis, a precentral distance effect was found in two subjects in the fast event-related fMRI paradigm, and such a location was also suggested by dipole modeling of the ERP distance effect. Moreover, previous studies using Arabic numerals also reported implication of the left precentral gyrus in various numerical tasks (Chochon et al., 1999; Pinel et al., 1999; Stanescu-Cosson et al., 2000; Pesenti et al., 2000; Zago et al., 2001). However, we found that an additional area of the precentral gyrus showed a notation effect with greater activation for verbal than for Arabic notation (Table 1) at coordinates consistent with previous studies of word reading (Fiez et al., 1999; Fiez and Petersen, 1998). Further research should clarify whether the contribution of this area to number processing is verbal or semantic.

\section{N umber Identification Processes}

Contrasting Arabic and verbal trials allowed us to examine the cerebral bases of the number identification systems that precede semantic access. The smaller P1 and greater extrastriate activity to verbal numerals than to Arabic numerals probably relate to superficial differences such as the greater size, complexity, and retinal eccentricity of the verbal numerals. More interesting is the finding of a greater right-lateralized N1 and greater right fusiform activation to Arabic numerals, replicating an earlier brief report (Pinel et al., 1999). Neuropsychological observations indicate that patients with lesions of the left visual system, and who are unable to read or compare verbal numerals, may show a perfect performance in comparing single- and two-digit Arabic numerals (Cohen and Dehaene, 1995, 2000b), suggesting that they have at their disposal a second route for identifying Arabic numerals. Because patients with a callosal disconnection can compare Arabic numerals presented in the left hemifield (Cohen and Dehaene, 1996; Seymour et al., 1994), this second route has been postulated to involve the right-hemispheric visual system (Dehaene and Cohen, 1995). The present results now provide direct evidence that the right fusiform gyrus is indeed implicated in the identification of Arabic numerals. 
A large-scale bilateral inferior parietal and frontal network also showed greater activation to Arabic than to verbal numerals. Those areas might relate to the different lexical and syntactic systems involved. Arabic notation is a positional system in which the same digital symbols take on a different meaning and can be expressed with different words depending on their location in the string (e.g., compare the digit 2 in 32, 12, and 20). The finding of specific impairments of Arabic number comprehension and transcoding (Cipolotti et al., 1995; Deloche and Seron, 1982, 1987; Noel and Seron, 1993) provides evidence for the existence of dedicated Arabic decoding mechanisms. Conversely, verbal numerals are amenable to a grapheme-to-phoneme reading route which is not available for Arabic numerals. An automatic activation of reading processes may explain our finding of greater activation of the left precentral sulcus by verbal than by Arabic numerals, at coordinates close to those found during other studies of word reading (Fiez et al., 1999; Fiez and Petersen, 1998). Indeed, the coordinates of this activation coincide with the site of the lesion in a patient with severe alexia and agraphia for written verbal material, but who could still read and write Arabic numerals perfectly (Anderson et al., 1990).

How is digit or word identity information transmitted from the areas involved in stimulus identification to the parietal quantity system? According to one model, "convergence zones" in the temporal lobes tie together conceptual and word-form information (Damasio, 1989; Damasio et al., 1996). In our experiment, a right middle temporal area was more active for Arabic than for verbal notation and showed a trend toward a distance $\times$ notation interaction, with a distance effect only in Arabic notation. The joint influence of both notation and semantic parameters on this region is consistent with its playing a role in the mediation between symbols and meanings. Specifically, this area might be involved in linking the right fusiform gyrus, which is involved in identifying Arabic numerals, with the parietal regions involved in notation-independent quantity coding. The contralateral left middle temporal region was also activated and showed a similar distance effect in both Arabic and verbal notation, consistent with a connection to the left fusiform region which is involved in identifying both notations (Dehaene, 1996). I nterestingly, Vandenberghe et al. (1996) showed that left temporal cortex showed an interaction of notation and semantic content when words were contrasted with pictures. It was activated more during semantic processing than during a nonsemantic control task, only when the concepts were presented verbally, not when they were presented as pictures. Such interactions are consistent with a role of temporal cortex in the mediation between symbols and meanings.

\section{CONCLUSION}

Using a factorial design, we isolated a network of coactivated bilateral parietal areas implicated in the semantic stage of the number comparison task. Similar activation patterns were observed for both Arabic and verbal notations in all these areas, independent of notation-induced changes in global task difficulty, thus reinforcing the assumption of a common format for quantity manipulation. Such parametric modulation supplements previous research on the modulation of parietal lobe activity by visuospatial factors and emphasizes the level of abstraction of the representations supported by this region. Fast event-related fMRI gave us the opportunity to detect those parietal foci in a single-subject analysis and to illustrate, at a finer scale, the strong correlation between parietal activity and behavioral performance.

\section{ACKNOWLEDGMENT}

This project was supported by the McDonnell Foundation.

\section{REFERENCES}

Aguirre, G. K., Detre, J . A., Alsop, D. C., and D’Esposito, M. 1996. The parahippocampus subserves topographical learning in man. Cereb. Cortex 6: 823- 829.

Anderson, S. W., Damasio, A. R., and Damasio, H. 1990. Troubled letters but not numbers. Domain specific cognitive impairments following focal damage in frontal cortex. Brain 113: 749-766.

Buckley, P. B., and Gillman, C. B. 1974. Comparison of digits and dot patterns. J . Exp. Psychol. 103: 1131-1136.

Caramazza, A. 1996. The brain's dictionary. Nature 380: 485- 486.

Carpenter, P. A., J ust, M. A., Keller, T. A., Eddy, W., and Thulborn, K. 1999. Graded functional activation in the visuospatial system with the amount of task demand. J . Cognit. Neurosci. 11: 9-24.

Chao, L. L., Haxby, J. V., and Martin, A. 1999. Attribute-based neural substrates in temporal cortex for perceiving and knowing about objects. Nat. Neurosci. 2: 913-919.

Chochon, F., Cohen, L., van de Moortele, P. F., and Dehaene, S. 1999. Differential contributions of the left and right inferior parietal lobules to number processing. J . Cognit. Neurosci. 11: 617-630.

Cipolotti, L., Butterworth, B., and Denes, G. 1991. A specific deficit for numbers in a case of dense acalculia. Brain 114: 2619-2637.

Cipolotti, L., Warrington, E. K., and Butterworth, B. 1995. Selective impairment in manipulating Arabic numerals. Cortex 31: 73- 86.

Cohen, L., and Dehaene, S. 1995. Number processing in pure alexia: The effect of hemispheric asymmetries and task demands. NeuroCase 1: 121-137.

Cohen, L., and Dehaene, S. 1996. Cerebral networks for number processing: Evidence from a case of posterior callosal lesion. Neurocase 2: 155-174.

Cohen, L., Dehaene, S., Naccache, L., Lehéricy, S., Dehaene-Lambertz, G., Hénaff, M. A., and Michel, F. 2000a. The visual word form area: Spatial and temporal characterization of an initial stage of reading in normal subjects and posterior split-brain patients. Brain 123: 291-307.

Cohen, L., and Dehaene, S. 2000b. Calculating without reading: Unsuspected residual abilities in pure alexia. Cognit. Neuropsychol. 17: 563-583. 
Corbetta, M., Akbudak, E., Conturo, T. E., Snyder, A. Z., Ollinger, J . M., Drury, H. A., Linenweber, M. R., Petersen, S. E., Raichle, M. E., Van Essen, D. C., and Shulman, G. L. 1998. A common network of functional areas for attention and eye movements. Neuron 21: 761-773.

Corbetta, M., Kincade, J. M., Ollinger, J. M., McAvoy, M. P., and Shulman, G. L. 2000. Voluntary orienting is dissociated from target detection in human posterior parietal cortex. Nat. Neurosci. 3: 292-297. [Published erratum appears in Nat. Neurosci., 2000, 3: 521]

Damasio, A. R. 1989. Time-locked multiregional retroactivation: A systems-level proposal for the neural substrates of recall and recognition. Cognition 33: 25- 62.

Damasio, H., Grabowski, T. J., Tranel, D., Hichwa, R. D., and Damasio, A. R. 1996. A neural basis for lexical retrieval. Nature 380: 499-505.

Dehaene, S. 1996. The organization of brain activations in number comparison: Event-related potentials and the additive-factors methods. J . Cognit. Neurosci. 8: 47- 68.

Dehaene, S., and Cohen, L. 1995. Towards an anatomical and functional model of number processing. Math. Cognit. 1: 83-120.

Dehaene, S., Dupoux, E., and Mehler, J . 1990. Is numerical comparison digital: Analogical and symbolic effects in two-digit number comparison. J . Exp. Psychol.: Hum. Percept. Perform. 16: 626- 641.

Dehaene, S., Spelke, E., Stanescu, R., Pinel, P., and Tsivkin, S. 1999. Sources of mathematical thinking: Behavioral and brain-imaging evidence. Science 284: 970-974.

Deloche, G., and Seron, X. 1982. From one to 1: An analysis of a transcoding process by means of neuropsychological data. Cognition 12: 119-149.

Deloche, G., and Seron, X. 1987. Numerical transcoding: A general production model. In Mathematical Disabilities: A Cognitive Neuropsychological Perspective (G. Deloche and X. Seron, Eds.), pp. 137-170. Erlbaum, Hillsdale, NJ .

Diwadkar, V. A., Carpenter, P. A., and J ust, M. A. 2000. Collaborative activity between parietal and dorso-lateral prefrontal cortex in dynamic spatial working memory revealed by fMRI. Neurol mage 12: 85-99.

Fiez, J. A., Balota, D. A., Raichle, M. E., and Petersen, S. E. 1999. Effects of lexicality, frequency, and spelling-to-sound consistency on the functional anatomy of reading. Neuron 24: 205-218.

Fiez, J. A., and Petersen, S. E. 1998. Neuroimaging studies of word reading. Proc. Natl. Acad. Sci. USA 95: 914-921.

Foltz, G. S., Poltrock, S. E., and Potts, G. R. 1984. Mental comparison of size and magnitude: Size congruity effects. J . Exp. Psychol.: Learn. 10: 442-453.

Ghaem, O., Mellet, E., Crivello, F., Tzourio, N., Mazoyer, B., Berthoz, A., and Denis, M. 1997. Mental navigation along memorized routes activates the hippocampus, precuneus, and insula. NeuroReport 8: $739-744$.

Gorno-Tempini, M. L., Price, C. J ., J osephs, O., Vandenberghe, R., Cappa, S. F., Kapur, N., and Frackowiak, R. S. 1998. The neural systems sustaining face and proper-name processing. Brain 121: 2103-2118.

Grön, G., Wunderlich, A. P., Spitzer, M., Tomczak, R., and Riepe, M. W. 2000. Brain activation during human navigation: Genderdifferent neural networks as substrate of performance. Nat. Neurosci. 3: 404-408.

Harris, I. M., Egan, G. F., Sonkkila, C., Tochon-Danguy, H. J., Paxinos G., and Watson, J . D. G. 2000. Selective right parietal lobe activation during mental rotation. A parametric PET study. Brain 123: $65-73$.

Hinrichs, J . V., Yurko, D. S., and Hu, J . M. 1981. Two-digit number comparison: Use of place information. J . Exp. Psychol.: Hum. Percept. Perform. 7: 890-901.
Kawashima, R., Naitoh, E., Matsumura, M., Itoh, H., Ono, S., Satoh, K., Gotoh, R., Koyama, M., Inoue, K., Y oshioka, S., and Fukuda, H. 1996. Topographic representation in human intraparietal sulcus of reaching and saccade. NeuroReport 7: 1253-1256.

Kosslyn, S. M., DiGirolamo, G. J ., Thompson, W. L., and Alpert, N. M. 1998. Mental rotation of objects versus hands: Neural mechanisms revealed by positron emission tomography. Psychophysiology 35: 151-161.

Le Clec'H, G., Dehaene, S., Cohen, L., Mehler, J ., Dupoux, E., Poline, J . B., Lehéricy, S., van de Moortele, P. F., and Le Bihan, D. 2000. Distinct cortical areas for names of numbers and body parts independent of language and input modality. Neurol mage 12: 381-391.

Mellet, E., Tzourio, N., Crivello, F., J oliot, M., Denis, M., and Mazoyer, B. 1996. Functional anatomy of spatial mental imagery generated from verbal instructions. J . Neurosci. 16: 6504-6512.

Menon, V., Rivera, S. M., White, C. D., Glover, G. H., and Reiss, A. L. 2000. Dissociating prefrontal and parietal cortex activation during arithmetic processing. Neurol mage 12: 357-365.

Morton, J . (1979). Word recognition. In Psycholinguistics, Ser. 2. Structures and Processes (J . Morton and J . Marshall, Eds.). Elek, London.

Noel, M. P., and Seron, X. 1993. Arabic number reading deficit: a single case study. Cognit. Neuropsychol. 10: 317-339.

Perani, D., Schnur, T., Tettamanti, M., Gorno-Tempini, M., Cappa, S. F., and Fazio, F. 1999. Word and picture matching: A PET study of semantic category effects. Neuropsychologia 37: 293-306.

Pesenti, M., Thioux, M., Seron, X., and De Volder, A. 2000. Neuroanatomical substrates of Arabic number processing, numerical comparison, and simple addition: A PET study. J . Cognit. Neurosci. 12: 461- 479.

Pinel, P., Le Clec'H, G., van de Moortele, P. F., Naccache, L., Le Bihan, D., and Dehaene, S. 1999. Event-related fMRI analysis of the cerebral circuit for number comparison. NeuroReport 10: 1473-1479.

Polk, T. A., and Farah, M. J. 1998. The neural development and organization of letter recognition: Evidence from functional neuroimaging, computational modeling, and behavioral studies. Proc. Natl. Acad. Sci. USA 95: 847-852.

Postle, B. R., Stern, C. E., Rosen, B. R., and Corkin, S. 2000. An fMRI investigation of cortical contributions to spatial and nonspatial visual working memory. Neurol mage 11: 409-423.

Richter, W., Somorjai, R., Summers, R., J armasz, M., Menon, R. S., Gati, J. S., Georgopoulos, A. P., Tegeler, C., Ugurbil, K., and Kim, S. G. 2000. Motor area activity during mental rotation studied by time-resolved single-trial fMRI.J . Cognit. Neurosci. 12: 310-320.

Seymour, S. E., Reuter-Lorenz, P. A., and Gazzaniga, M. S. 1994. The disconnection syndrome: Basic findings reaffirmed. Brain 117: 105-115.

Shallice, T. 1988. From Neuropsychologia to Mental Structure Cambridge Univ. Press, Cambridge, UK.

Shepard, R. N., and Metzler, J. 1971. Mental rotation of threedimensional objects. Science 171: 701-703.

Stanescu-Cosson, R., Pinel, P., van de Moortele, P.-F., Le Bihan, D., Cohen, L., and Dehaene, S. 2000. Understanding dissociations in dyscalculia: A brain imaging study of the impact of number size on the cerebral networks for exact and approximate calculation. Brain 123: 2240-2255.

Sternberg, S. 1969. The discovery of processing stages: Extensions of Donders' method. Acta Psychol. 30: 276-315.

Tagaris, G. A., Kim, S. G., Strupp, J . P., Andersen, P., U gurbil, K., and Georgopoulos, A. P. 1996. Quantitative relations between parietal activation and performance in mental rotation. NeuroReport 7: 775-776. 
Temple, E., and Posner, M. I. 1998. Brain mechanisms of quantity are similar in 5-year-olds and adults. Proc. Natl. Acad. Sci. USA 95: 7836-7841.

Thomas, K. M., King, S. W., Franzen, P. L., Welsh, T. F., Berkowitz, A. L., Noll, D. C., Birmaher, V., and Casey, B. J . 1999. A developmental functional MRI study of spatial working memory. NeuroI mage 10: 327-338.

Tzeng, O. J . L., and Wang, W. 1983. The first two R's. Am. Sci. 71: 238-243.
Vandenberghe, R., Price, C., Wise, R., J osephs, O., and Frackowiak, R. S. 1996. Functional anatomy of a common semantic system for words and pictures. Nature 383: 254-256.

Vogt, B. A., and Pandya, D. N. 1987. Cingulate cortex of the rhesus monkey. II. Cortical afferents. J. Comp. Neurol. 262: 271-289.

Zago, L., Pesenti, M., Mellet, E., Crivello, F., Mazoyer, B., and Tzourio-Mazoyer, N. 2001. Neural correlates of simple and complex mental calculation. Neurol mage 13: 314-327. 